\title{
VEGF-D is an X-linked/AP-1 Regulated Putative Onco-angiogen in Human Glioblastoma Multiforme
}

\author{
Waldemar Debinski, ${ }^{1}$ Becky Slagle-Webb, ${ }^{1}$ Marc G. Achen, ${ }^{2}$ Steven A. Stacker, ${ }^{2}$ Eugene Tulchinsky, ${ }^{3}$ \\ G. Yancey Gillespie, ${ }^{4}$ and Denise M. Gibo ${ }^{1}$ \\ ${ }^{1}$ Division of Neurosurgery/H110, Pennsylvania State University College of Medicine, Hershey, \\ Pennsylvania, USA \\ ${ }^{2}$ Ludwig Institute for Cancer Research, Royal Melbourne Hospital, Victoria, Australia \\ ${ }^{3}$ Department of Molecular Cancer Biology, Danish Cancer Society, Copenhagen, Denmark \\ ${ }^{4}$ Division of Neurosurgery, Department of Surgery, The University of Alabama at Birmingham, School of \\ Medicine, Birmingham, Alabama, USA \\ Accepted July 10, 2001
}

\begin{abstract}
Background: Glioblastoma multiforme (GBM) is a hypervascularized and locally infiltrating brain tumor of astroglial origin with a very poor prognosis. An X-linked c-fos oncogene-inducible mitogenic, morphogenic, and angiogenic factor, endothelial growth factor-D (VEGF-D), is the newest mammalian member of VEGF family. We analyzed VEGF-D in GBM because of its high angiogenic potential and its linkage to the $\mathrm{X}$ chromosome.

Materials and Methods: Nonmalignant brain and GBM tissue sections as well as GBM cell lines were analyzed by immunofluorescence for the expression of VEGF-D, factor VIII (endothelial cell marker), glial-fibrillary acidic protein (GFAP) (astrocytic cell lineage cytoplasmic marker), and several Fos family transcription factors, including c-Fos and Fra-1. The proteins were also detected by Western blots. The differences between genotypes of normal brain and GBM cells were examined by cDNA microarrays.
\end{abstract}

Results and Conclusions: GBM expressed ubiquitously VEGF-D, which colocalized with GFAP. Contrary to our expectations, low levels of c-Fos were detected in GBM cells. However, we identified another Fos family member, Fra-1, together with its transcriptional activation partner, c-Jun, as being stably up-regulated in GBM cells. Furthermore, we demonstrated that a fra- 1 transgene induced VEGF-D expression in cultured cells and GBM cell stimulation evoked a sustained increase in both Fra-l and VEGF-D levels. This study reveals that an up-regulation of AP-1 factors may be a hallmark of GBM. Because VEGF-D activates VEGF receptor 2 and 3, receptors important for tumor angiogenesis, it may represent an X-linked/ AP-1-regulated onco-angiogen in human GBM. The VEGF-D system and AP-1 activity appear to be very attractive targets for new molecular diagnostics and rational molecular anti-cancer therapies.

\section{Introduction}

Glioblastoma multiforme (GBM), a human brain malignancy originating from glial non-neuronal cells, is characterized by a well-developed and abnormal vascularization (1). An increase in endothelial cell proliferation and the subsequent collapse of some areas of vasculature with following focal necrosis are diagnostic characteristics useful in differentiating GBMs from other brain tumors. In particular, the endothelium lining the vessels in GBM is profoundly morphologically different from normal brain endothelium (2). Previous research implicated vascular endothelial growth factor (VEGF-A), a member of the platelet-derived growth factor/VEGF family of

Address correspondence and reprint requests to: Waldemar Debinski, MD, PhD, Director of Tumor Research, Section of Neurosurgery/H110, Department of Surgery, Pennsylvania State University College of Medicine, 500 University Drive, Hershey, PA 17033-0850. Phone: (717) 531-4541; fax: (717) 531-5906; e-mail: wdebinski@psu.edu polypeptide growth factors, in this brain tumor pathogenesis because it is an oncogene-activated angiogenic factor $(3,4)$. Recently, the newest mammalian member of this family was identified and designated VEGF-D $(5,6)$. VEGF-D binds and activates VEGF receptor 2 (Flk-1/KDR) and VEGF receptor 3 (Flt-4), but not VEGF receptor 1 (Flt-1) (6). The binding and signaling activity of the first preparations of VEGF-D were similar to that of VEGF-A, although it was several times less potent (6). VEGF-D is mitogenic for endothelial cells, dramatically changes the morphology of endothelial cells, and promotes angiogenesis $(6,7)$.

In a search for GBM-specific markers, we previously discovered large amounts of a receptor for interleukin 13 (IL-13), an immune regulatory cytokine, in a vast majority of patients with GBM in situ (8). This receptor is a "restrictive" receptor for IL-13, because it does not bind IL-4, a homolog of IL-13, in contrast to the normal tissue receptor, IL-13/-4R, that binds both IL-13 and IL-4 $(9,10)$. The GBM-associated IL-13R 
has been found primarily in brain tumors of glial, non-neuronal, origin (11) and it is a single chain 42$\mathrm{kDa}$ plasma membrane receptor, termed IL-13R $\alpha 2$ (Mintz et al., submitted). We recently identified IL13R $\alpha 2$ as a cancer/testis tumor antigen (CTA) (10). CTAs constitute a growing group of tumor antigens, the genes of which reside on chromosome X (12). We thus became interested in a possibility of X-linked factors being involved in the pathogenesis of GBM (13).

The gene for VEGF-D resides on chromosome $\mathrm{X}$ (14). Importantly, VEGF-D was isolated as a c-fos oncogene-inducible mitogen (5). Because VEGF-D has the potential to induce tumor angiogenesis, represents an oncogene-inducible factor, and is X-linked, we decided to explore VEGF-D in highly vascularized and highly malignant GBM tumors. Our study demonstrates a ubiquitous presence of VEGF-D in GBM owing to an up-regulation of AP-1 factors other than c-Fos in the diseased cells. This phenomenon was uncovered with the help of cDNA microarray analyses of normal and malignant central nervous system tissues.

\section{Materials and Methods}

Cell Lines and Tissues

GBM cells lines A-172 MG, U-251 MG, DBTRG-50 MG, U-87 MG, U-373 MG, human GBM explant cells, G48a (isolated in our laboratory), CSML0, and CSML100 mouse breast carcinoma cells were grown in the appropriate media. The CSMLO and CSML100 mouse breast cancer cells and GBM Al72 MG glioblastoma cells were grown in Dulbecco's Modified Eagle's Medium (D-MEM) with 10\% fetal calf serum (FCS) (Life Technologies, Rockville, MD, USA). U-251 MG cells were grown in D-MEM, 10\% FCS, $0.1 \mathrm{mM}$ minimum essential medium (MEM) nonessential amino acids (NEAA) (Life Technologies), and $50 \mu \mathrm{g} / \mathrm{ml}$ gentamicin sulfate. GBM cell lines U-87 MG and U-373 MG were grown in Earle's MEM, 10\% FCS, $0.1 \mathrm{mM}$ NEAA, $2 \mathrm{mM}$ glutamine (Life Technologies), and $100 \mu \mathrm{g} / \mathrm{ml}$ sodium pyruvate. GMB cell line DBTRG-50 MG and human explant cells were grown in RPMI-1640 (Life Technologies), $10 \%$ FCS, $100 \mu \mathrm{g} / \mathrm{ml}$ sodium pyruvate, $100 \mu \mathrm{g} / \mathrm{ml}$ L-cystine (Life Technologies), $20 \mu \mathrm{g} / \mathrm{ml}$ L-proline (Sigma, St. Louis, MO, USA), Ix HT supplement, consisting of $0.1 \mu \mathrm{M}$ sodium hypoxanthine and $0.016 \mu \mathrm{M}$ thymidine, $5 \mathrm{U} / \mathrm{ml}$ penicillin $\mathrm{G}$ and $5 \mathrm{U} / \mathrm{ml}$ streptomycin sulfate (Penn/ Strep, Life Technologies). Normal human astrocyte cells (NHA) were grown in Astrocyte Growth Medium BulletKit (BioWhittaker, Walkersville, MD). Normal human umbilical vein endothelial cells (HUV-EC-C) were grown in F-12 Kaighn's medium (Life Technologies) with $10 \%$ FCS, $100 \mu \mathrm{g} / \mathrm{ml}$ heparin (Sigma), and $30 \mu \mathrm{g} / \mathrm{ml}$ Endothelial Cell Growth Supplement (Sigma).

A retroviral vector was used to generate plasmid pMVfra-1 (Mahmood et al., manuscript submitted). To produce replication-defective retroviruses, the $\mathrm{GP}+\mathrm{E}$ packaging cell line was employed, which was maintained in appropriate media. Successfully transfected GP+E cells were selected in the presence of $800 \mu \mathrm{g} / \mathrm{ml} \mathrm{G} 418$. Supernatants of virus-producing cell lines were used to infect CSMLO cells. Infected cells were selected in the presence of $400 \mu \mathrm{g} / \mathrm{ml} \mathrm{G} 418$.

GBM tumors and nonmalignant brain tissue, the latter obtained usually from the therapeutic resections for the treatment of epilepsy, were obtained from the operating room and snap frozen immediately, as described previously (8). Ten-micron sections of GBM were thaw mounted onto chrom-alum slides. Slides were stored at $-80^{\circ} \mathrm{C}$ until assayed. Sections were allowed to thaw and subsequently fixed for $10 \mathrm{~min}$ in acetone at $-20^{\circ} \mathrm{C}$.

\section{Immunostaining}

GBM cells lines, human explant cells (G48a), HUVEC-C from ATCC (Rockville, MD, USA) and NHA from BioWhittaker were grown overnight on sterile glass slides in the appropriate media. Slides were washed twice in phosphate-buffered saline (PBS) and fixed for $2 \mathrm{~min}$ in acetone at $-80^{\circ} \mathrm{C}$. Slides were washed twice in PBS and either used immediately or air dried and stored at $-80^{\circ} \mathrm{C}$ until assayed. In stimulation experiments, $10^{4}$ SNB-19 GBM cells were plated on glass chamber slides and allowed to attach overnight. The cells were washed with PBS and serum-free media was applied.

After $24 \mathrm{hr}$ epidermal growth factor (EGF) or leukemia inhibitory factor (LIF) were added to cells at 20 and $5 \mathrm{ng} / \mathrm{ml}$, respectively. The cells were processed for immunocytochemistry after the 24-hr stimulation period.

Mouse monoclonal anti-VEGF-D (VD1) antibody was used (15). It was employed at a final dilution of 1:500 $(7.5 \mu \mathrm{g} / \mathrm{ml})$. Other primary antibodies including rabbit polyclonal Fra-1 (1:100), c-Fos (1:100), c-Jun (1:150), and mouse monoclonal JunB (1:75) were purchased from Santa Cruz Biotechnology (Santa Cruz, CA, USA); and mouse monoclonal factor VIII (1:150) and rabbit glial-fibrillary acidic protein (GFAP) (1:500) were purchased from DAKO Chemical (Carpinteria, CA, USA).

Slides were washed in two changes of PBS and blocked for 30 min with $10 \%$ (v:v) normal goat serum (NGS) in PBS at room temperature. Primary antibody was diluted in $1.5 \%$ NGS/PBS and incubated at room temperature for either $1 \mathrm{hr}$ (VEGF-D, factor VIII, and GFAP) or 2 hr (Fra-1, JunB, c-Fos, and c-Jun). Slides were washed in three changes of PBS for 10 min each. Secondary antibody, goat antirabbit Rhodamine (1:150) (Jackson ImmunoResearch Laboratories, Inc., West Grove, PA, USA) or sheep anti-mouse Cy3 (1:250), (Sigma) was diluted in $1.5 \% \mathrm{NGS} / \mathrm{PBS}$ and incubated in the dark at room temperature for $45 \mathrm{~min}$. For double-labeling experiments, the secondary antibodies were goat anti-mouse Oregon GreenR (1:200) (Molecular Probes, Eugene, OR, USA) and goat anti-rabbit Rhodamine (1:150). Slides were washed in three 
changes of PBS for 10 min each and mounted with Gel-Mount (Biomeda Corp., Foster City, CA, USA). Some slides were counterstained with Hoechst No. 33258 Nuclear Counterstain (DAPI).

Photomicrographs were taken at $40 \times$ magnification in all cases with a Hamamatsu C2400 digital camera. Background was normalized to the samples without primary antibody. Each set of images was taken exactly at the same exposure settings. Images were processed with Paint Shop Pro V 6.0 (Jasc Software Inc., Eden Prairie, MN, USA).

\section{Western Blots}

Cell lysates were prepared from subconfluent cultures. Cells were washed twice in PBS and lysed in RIPA buffer (PBS, 1\% Igepal CA-630; ICN Biomedicals, Inc. Costa Mesa, CA, USA), $0.5 \%$ sodium deoxycholate (Fisher Scientific, Fair lawn, NJ, USA), $0.1 \%$ SDS-containing Mammalian Protease Inhibitor Cocktail (Sigma). GBM and nonmalignant brain tumor samples were minced into small pieces while frozen and thawed in RIPA buffer with Mammalian Protease Inhibitor Cocktail. Lysates were passed through a 21-gauge needle to shear the DNA. PMSF (1 $\mathrm{mM}$, Sigma) was added and the lysates were incubated on ice for 30-60 min. Nonsolubilized debris was pelleted at $10,000 \times \mathrm{g}$ for $10 \mathrm{~min}$. The supernatant was collected, aliquoted, and stored at $-80^{\circ} \mathrm{C}$ until use. Normal human brain lysates were also purchased from Chemicon International, Inc. (Temecula, CA, USA) and Clontech Laboratories, Inc. (Palo Alto, CA, USA).

Lysates were run on either $12 \%$ or $15 \%$ SDSPAGE. Proteins were transferred to PVDF membrane (Pierce, Rockford, IL, USA) and blocked for $1 \mathrm{hr}$ with $5 \%$ blotto ( $5 \%$ dry milk, PBS, $0.05 \%$ Tween-20). Membranes were incubated with primary antibody diluted in blotto for $40 \mathrm{~min}$ at room temperature while shaking. Antibodies included anti-mouse VEGF-D antibody (40\% cross-reactivity with human VEGF-D; 1:500) from R\&D Systems, Minneapolis, MN and Fra-1 (1:100) from Santa Cruz Research Antibodies. Following three 5-min washes in PBS $/ 0.05 \%$ Tween-20, membranes were incubated in secondary antibody conjugated with horseradish peroxidase (goat anti-mouse IgG or goat anti-rabbit IgG) at a dilution of $1: 10,000$ or $1: 15,000$ in $5 \%$ blotto for $40 \mathrm{~min}$ at room temperature while shaking. Membranes were washed in several changes of PBS and detection was performed using the SuperSignal West Pico Chemiluminescent Substrate (Pierce). Membranes were exposed to autoradiographic film X-OMAT AR for up to $5 \mathrm{~min}$. Films were scanned in a transparency scanner at a pixel size of $88 \times 88 \mathrm{mi}-$ cron (Molecular Dynamics, Sunnyvale, CA, USA). The images were compiled in Paint Shop Pro V 6.0.

\section{cDNA Arrays}

Atlas Oncogene/Tumor Suppressor Arrays were purchased from Clontech and $1 \mu \mathrm{g}$ of poly(A) + RNA was labeled with $\left[\alpha-{ }^{33} \mathrm{P}\right] \mathrm{dATP}$ according to the manufacturer. Membranes were prehybridized overnight at $68^{\circ} \mathrm{C}$ in ExpressHyb (Clontech) containing $0.1 \mathrm{mg} /$ $\mathrm{ml}$ sheared salmon sperm DNA. Labeled cDNA probe was denatured and added to the prehybridization solution and the membranes were hybridized overnight at $68^{\circ} \mathrm{C}$. Membranes were then washed twice in $2 \times \mathrm{SSC} / 1 \%$ SDS for $20 \mathrm{~min}$ followed by two washes in $0.1 \%$ SSC $/ 0.5 \%$ SDS at $68^{\circ} \mathrm{C}$. The membranes were exposed to autoradiographic film for up to 10 days at $-70^{\circ} \mathrm{C}$. The arrays contain cDNA-specific fragments for oncogenes, such as $c$-fos, junB, and $c-m y c$. Housekeeping genes included ubiquitin, liver glyceraldehyde 3-phosphate dehydrogenase, and phospholipase. RNA used for the cDNA micro-array assays was isolated from subconfluent cultures of GBM cells using the acid-guanidium isothiocyanate-phenolchloroform method (16). Poly(A) + RNA was further isolated using the Oligotex mRNA Kit (Qiagen Inc, Valencia, CA, USA). Normal human brain Poly(A)+ RNA was purchased from Clontech.

\section{Karyotyping}

The karyotypes of HGA cells analyzed in this study were performed in a blinded fashion by clinical cytogeneticists at the Cancer Genetics Laboratory, Genetics \& IVF Institute, Fairfax, Virginia, USA.

\section{Results}

Normal Brain Immunoreactivity for VEGF-D

To establish the expression of VEGF-D in normal brain, we performed immunofluorescence studies using sections of nonmalignant brain tissue. In a previous analysis of gene expression among a series of normal organs, whole human brain demonstrated a very low level of transcripts for VEGF-D $(6,17)$. Using 10 randomly selected specimens of human brain, we found that some immunoreactivity could be detected in normal brain using an anti-VEGF-D antibody VDl (15) (Fig. 1A). Some tissue sections of nonmalignant brain showed a weak staining for VEGF-D, which was localized primarily to the cell cytoplasm and cell processes (e.g., Fig. 1A, NB-1, -3, -5, and -7). The remaining samples showed sporadic and even poorer staining, if at all. On the contiguous sections of the same piece of nonmalignant brain, the staining of endothelial cell-associated von Willebrand factor (factor VIII) was evident, scattered and corresponded to the expected picture of vessel density and size seen in nonmalignant brain (Fig. 1A). Small caliber vessels imprinted by the staining with the antibody against factor VIII were seen in all sections (Fig. 1A). The background fluorescence in these experiments (i.e., without primary antibody added) was low (Fig. 1A, "no primary antibody" panel).

\section{VEGF-D Is Abundant in GBM}

The presence of VEGF-D has never been analyzed in brain tumors. Using exactly the same conditions as 
A

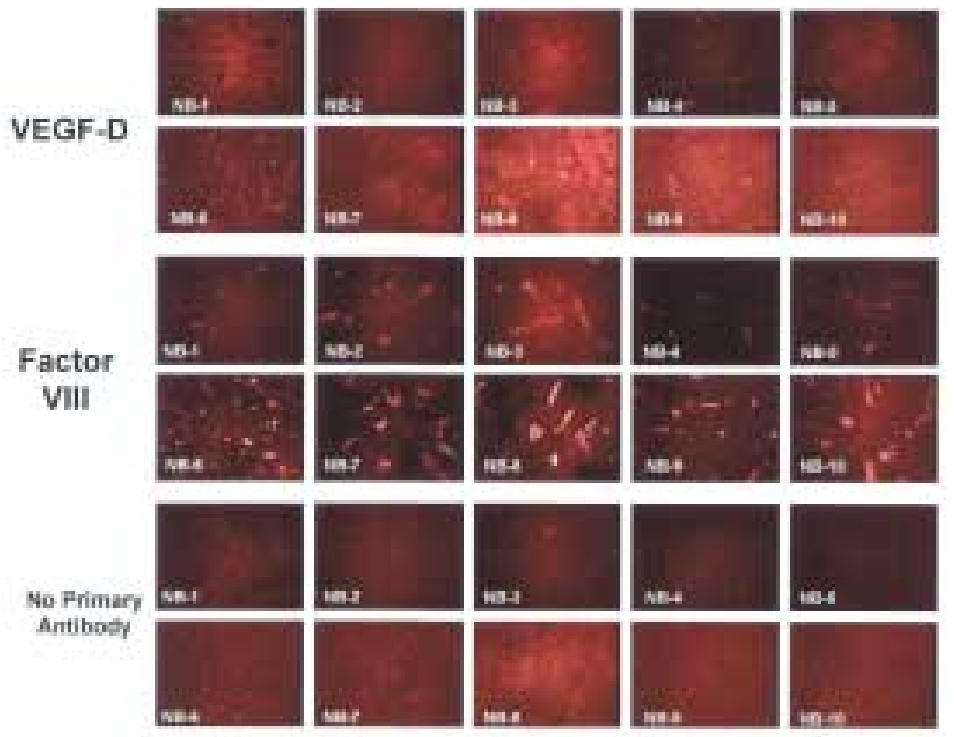

B
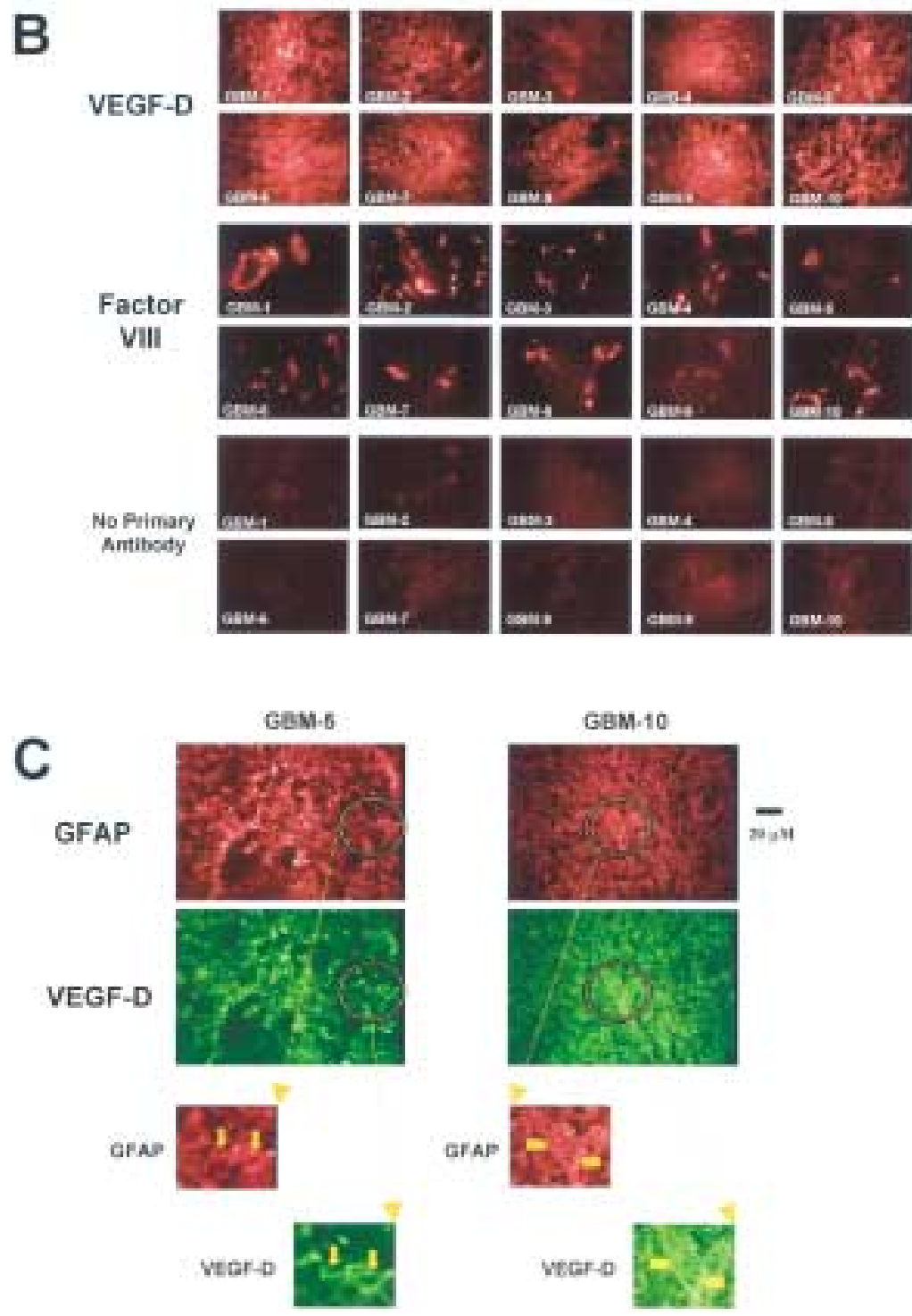
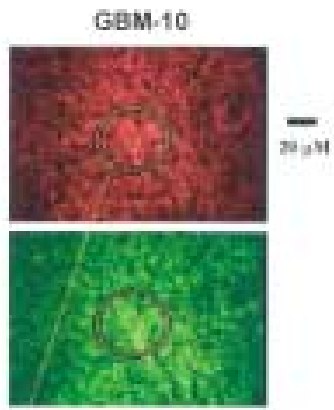

Grap

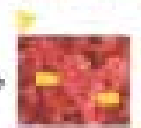

vecro
D factor VIII immunofluorescence in contiguous sections of 10 different tissue specimens of nonmalignant human brain (NB). The bottom two rows of tissue sections represent a control assay in which no anti-VEGF-D or anti-factor VIII antibody was added. (B) VEGF-D and factor VIII immunofluorescence in contiguous sections of 10 different specimens of human GBM. The bottom two rows of tissue sections represent a control assay, as in (B). (C) Double staining of GBM specimens with glialfibrillary acidic protein (GFAP) (red) and VEGF-D (green). The sections of staining for GFAP and VEGF-D correspond to a proportionate enlargement of the sections as shown in $\mathbf{A}$ and $\mathbf{B}$. Circled areas were again enlarged $2 \times$. Arrows point to cell bodies and pentagons point to astrocytoma cell processes. (D) Triple staining of a GBM specimen with GFAP (red), VEGF-D (green), and DAPI, a nuclear counter-stain (blue).
GFAP

VEGF-D

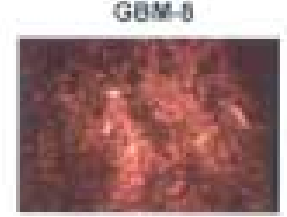

DAPI

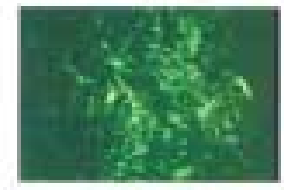

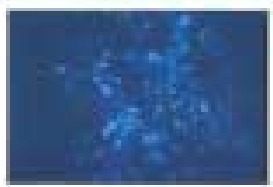


for immunofluorescence studies in nonmalignant brain, we discovered a distinctively different picture of VEGF-D staining in malignant brain tissues (Fig. 1B). GBM sections demonstrated high levels of immunofluorescence for VEGF-D when compared with nonmalignant brain and controls (Fig. 1B). VEGF-D showed a pattern of staining most compatible with cytoplasmic localization (i.e., diffused within the malignant astrocytic cells and also concentrated in their processes). All 10 randomly selected tumors showed this pattern, suggesting a remarkably high prevalence rate of VEGF-D overproduction/accumulation in GBMs.

GBMs are known to be highly vascular, which can be demonstrated by staining an antigen inherent to endothelial cells. Therefore, an antibody against factor VIII was used in parallel immunofluorescence studies with tissue specimens; it was isotype matched to the anti-VEGF-D antibody VDl. Antifactor VIII antibody immunofluorescence staining showed a characteristic pattern of hyperplastic vessels present on contiguous tissue sections of the same GBM as used for VEGF-D staining (Fig. 1B). Factor VIII staining demonstrated larger and more complex blood vessels in GBM tissue than seen in nonmalignant brain (Fig. 1B).

The pattern of VEGF-D staining in GBM was strongly suggestive of astrocytoma cells expressing this angiogenic factor (Fig. 1B). To document this directly, we performed a double-staining experiment using several GBM specimens, three of which are shown in Figure IC and D. We stained GBM sections for GFAP, which is the most specific cytoplasmic marker for astrocytoma cells available (18). GFAP staining of GBM revealed a typical cytoplasmic localization that outlines both astrocytoma cell bodies and their at times tube-like processes (Fig. 1C/1D, red color). VEGF-D staining colocalized with the staining for GFAP (Fig. 1C/1D, green color). In Figure 1D, GBM specimen 8 was stained with DAPI, a nuclear counterstain, which further contrasted the cytoplasmic localization of VEGF-D. Thus, we established a prominent presence of VEGF-D in the cytosol of astrocytoma cells of GBM in situ.

\section{Astrocytoma Cells Synthesize VEGF-D}

The pattern of VEGF-D staining in GBM tissue specimens indicated that the tumor cells, astrocytoma cells, were the principal source of in situ detected VEGF-D (Figs. 1B/1C). To document that astrocytoma cells in fact express VEGF-D, we analyzed several GBM cell lines for both VEGF-D and factor VIII immunoreactivity (Fig. 2A). Six tumor cell lines studied showed a prominent immunofluorescent staining for VEGF-D (Fig. 2A). GBM cells, such as A-172 MG, G48a, U-87 MG, DBTRG-50 MG, U-25 I MG, and U-373 MG, demonstrated a diffuse cytoplasmic staining pattern (Fig. 2A). This diffuse cytoplasmic pattern provided a characteristic nuclear outline generated by VEGF-D (Fig. 2A). In contrast, the analysis of the nonmalignant cell lines, HUV-EC-C, and NHAs, demonstrated low levels of immunoreactivity for VEGF-D, although this was more pronounced in NHA than in HUV-EC-C (Fig. 2A, right panel). Furthermore, HUV-EC-C stained prominently for factor VIII in the cytoplasm and was the only cell line positive for this endothelial cell marker used in the experiment (Fig. 2A). Background fluorescent staining was low in all specimens and cell lines examined when using an irrelevant mouse $\operatorname{IgG}_{1}$ or no primary antibody (Fig. 2B).

VEGF-D is an X-linked factor and therefore we analyzed the karyotypes of astrocytoma cells used in our experiments. As shown in Figure 2A (upper panel with staining for VEGF-D), five out of six established cell lines exhibited abnormal ploidy for chromosome $\mathrm{X}$, the most frequent alteration being an additional chromosome X.

The high content of VEGF-D immunoreactivity in GBM tumor specimens and GBM cell lines indicated VEGF-D protein therein. Cell lysates of four of the cell lines were analyzed by SDS-PAGE and Western blotting performed with the anti-VEGF-D antibody. A single protein band was detected with an approximate molecular weight of $48 \mathrm{kDa}$ (Fig. $2 \mathrm{C})$. This protein band corresponds to the fulllength VEGF-D (19). The GBM cell lines U-25 1 MG and DBTRG-50 MG possessed this protein, but it was not found in the lysates from either the astrocyte (NHA) or endothelial (HUV-EC-C) cell lines. These data, taken together with the specific immunostaining of tissues and cells, indicate that GBM tumor cells produce abundant amounts of VEGF-D, an X-linked angiogenic factor.

\section{The Fos Transcription Factors in GBM Cells}

VEGF-D has been reported as a c-fos-inducible mitogenic and morphogenic factor, and named accordingly a c-fos-induced growth factor (5). It was thus imperative to explore the c-fos oncogene protein expression in GBM, because it was the foremost suspect responsible for high and ubiquitous overexpression of VEGF-D. Contrary to expectations, the levels of c-Fos in several GBM cell lines were low in our assays (Fig. 3A). Specific nuclear immunoreactivity for c-Fos was seen mainly in some of the DBTRG-50 MG cells (Fig. 3A). Others found similarly low levels of c-fos gene expression in brain tumor cells (20). Thus, factors other than c-Fos may be involved in sustained VEGF-D up-regulation in GBM cells.

The gene for VEGF-D has an optimal AP-1 binding site in its promoter region (17). Considering the lack of correlation between the levels of c-Fos and VEGF-D in GBM cells, we looked into the possibility that other AP-1 transcription factors, to which c-Fos belongs, play roles in VEGF-D up-regulation. Our experiments with oncogene/tumor suppressor gene-containing cDNA microarrays revealed that the expression of the fos-related antigen-1 gene (fra- 1$)$ is higher in GBM cells, such as G48a (Fig. 3B; dots i-9), 


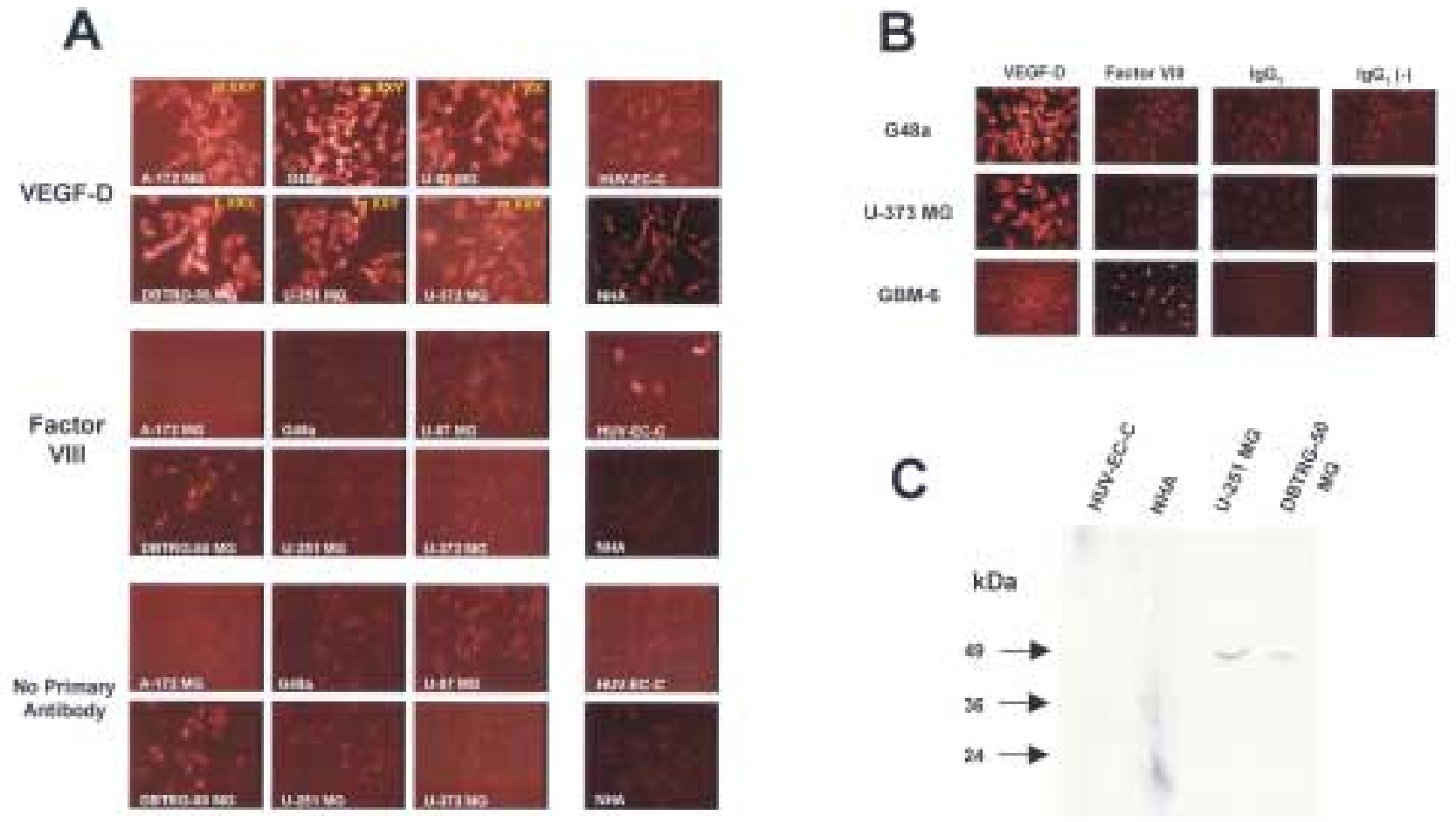

Fig. 2. VEGF-D immunoreactivity in GBM cells. (A) VEGF-D and factor VIII immunofluorescence analyzed in six different GBM cell lines and two normal cell lines: endothelial cell line, HUV-EC-C, and astrocytic cells (NHA). Cell designation is given in the left-bottom corners of the panels (white) and their karyotype is given in the right-top corners of the panels (yellow). $f$, female; $m$, male. The bottom two rows represent a control assay in which no anti-VEGF-D or anti-factor VIII antibody was added. (B) Control immunostaining of GBM cells

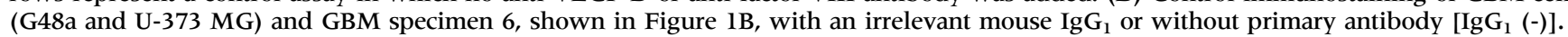
(C) Western blot immunoreactivity for VEGF-D in cell lysates of human GBM. Equal amounts (40 $\mu \mathrm{g} / \mathrm{lane})$ of cell lysate proteins derived from U-251 MG and DBTRG-50 MG GBM cells, and HUV-EC-C and NHA cells were electrophoretically separated under reducing conditions and blotted onto a PVDF membrane. The immunoreactivity was developed using chemiluminescence.

when compared with normal brain tissue, whereas the expression of c-fos was usually undetectable (Fig. 3B; dots b-19). The same phenomenon was observed in DBTRG-50 MG and U-87 MG GBM cell lines (data not shown). Therefore, we performed immunofluorescence using anti-Fra-l antibody in GBM cells. We discovered that Fra- 1 is highly expressed in all GBM cell lines studied, such as G48a, U-87 MG, U251 MG, and DBTRG-50 MG (Fig. 3C). However, the pattern of staining for Fra-1 was distinctly different from the rather diffuse cytoplasmic staining for VEGF-D (Fig. 2A). Anti-Fra-1 immunopositivity was localized to the nuclei of the cells, which would be expected for this transcription factor. HUV-EC-C demonstrated limited nuclear immunofluorescence for Fra-1 (Fig. 3C). We performed Western blots on GBM cell lysates and tumor samples and found a protein band of $\sim 42 \mathrm{kDa}$, which corresponds to the size of human Fra-1 (data not shown).

Fra-1 cannot activate gene expression itself; it requires heterodimerization with Jun proteins to do so. c-Jun and JunB in particular are preferable partners for Fra-1 and, in the process of Fra-1 up-regulation in response to Ras activation, c-Jun was primarily utilized as the binding partner with Fra-1 (21). Based on cDNA microarray analyses we found $c$-jun, and much less junB, expressed in astrocytoma cells (Fig. 3B; dots $\mathrm{k}-4$ for $c$-jun and dots $\mathrm{d}-12$ for junB). In follow-up immunohistochemical studies, the staining for c-Jun was readily detected and localized to the nuclei of GBM cells, similar to the Fra-1 staining (Fig. 3D). JunB was detected by immunohistochemistry, although at lower intensities than c-Jun (Fig. 3B). Interestingly, $c$-jun was the only gene expressed in normal brain tissue among AP-1 factors (Fig. 3B, "Normal Brain" panel; dots k-4).

\section{Fra-1 Induces Expression of VEGF-D}

Our current investigation focused our attention on Fra1 and its role in transcriptional activation of other factors, likely suspects involved in cancer progression/ maintenance. For example, Fra-1 has been previously identified as the primary AP-1 factor involved in the development of a more invasive, highly progressive carcinoma phenotype of breast cancer (22). We thus performed immunoblot for VEGF-D in cell lysates of CSML0 (low Fra-1) and CSML100 (high Fra-1) mouse breast cancer cells (22) (Fig. 4A). We found an elevated VEGF-D in CSML100 when compared with CSMLO cells (Fig. 4A). Furthermore, we used mocktransfected and fra-1-transfected CSMLO cells. The mock-transfected CSML0 cells did not express Fra-1, 


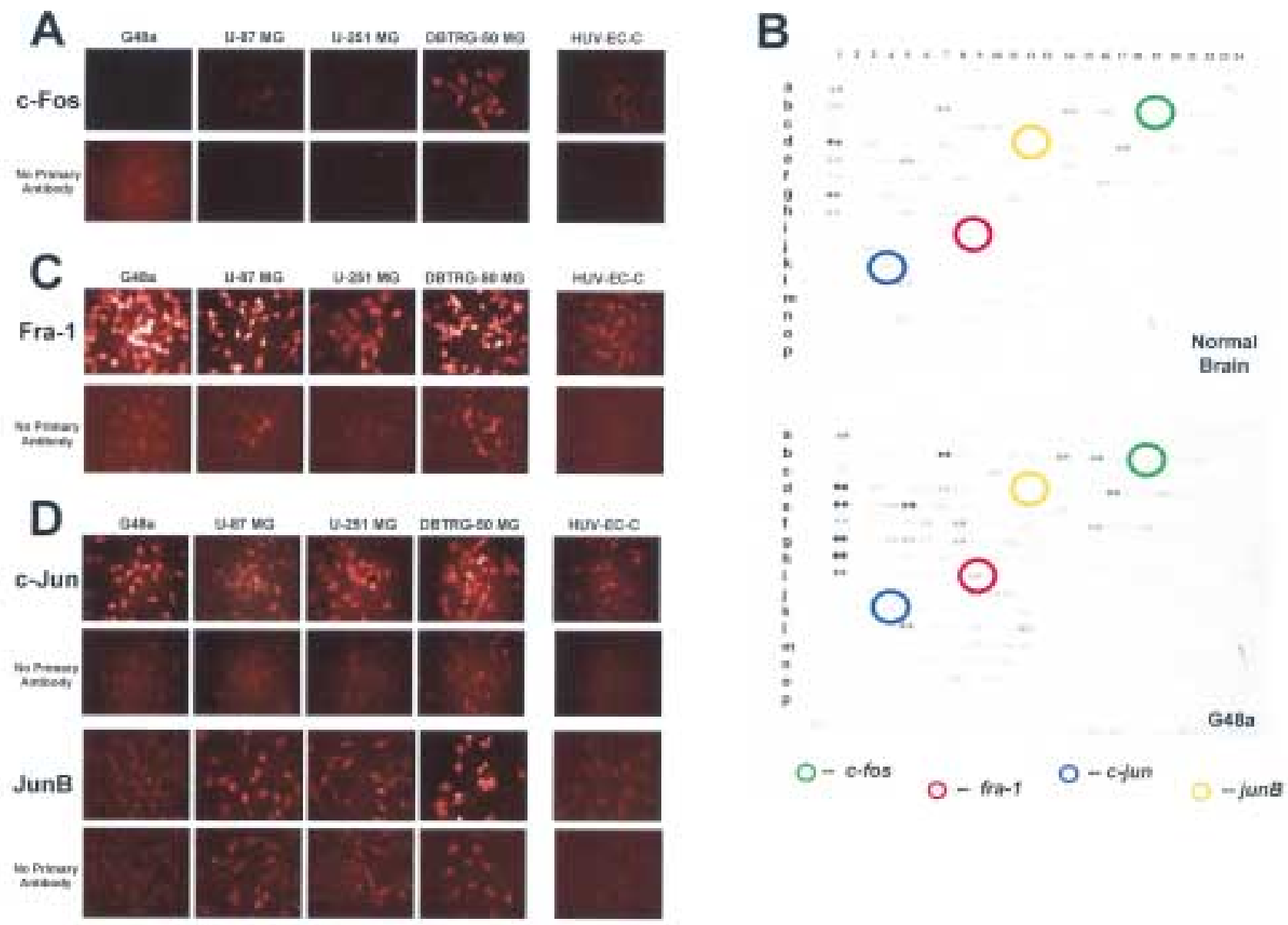

Fig. 3. Fos/Jun immunoreactivity and gene expression in human GBM cells. (A) c-Fos immunofluorescence examined in four GBM cell lines (G48a, U-87 MG, U-251 MG, and DBTRG-50 MG) and HUV-EC-C. (B) cDNA microarray analysis of the genes expressed in normal brain and G48a GBM cells. Atlas Human Oncogene/Tumor Suppressor Arrays (Clontech) membranes were used. (C) Fra-1 immunofluorescence in GBM cell lines and normal endothelial cells, HUV-EC-C. (D) c-Jun and JunB immunofluorescence in GBM cell lines and HUV-EC-C.
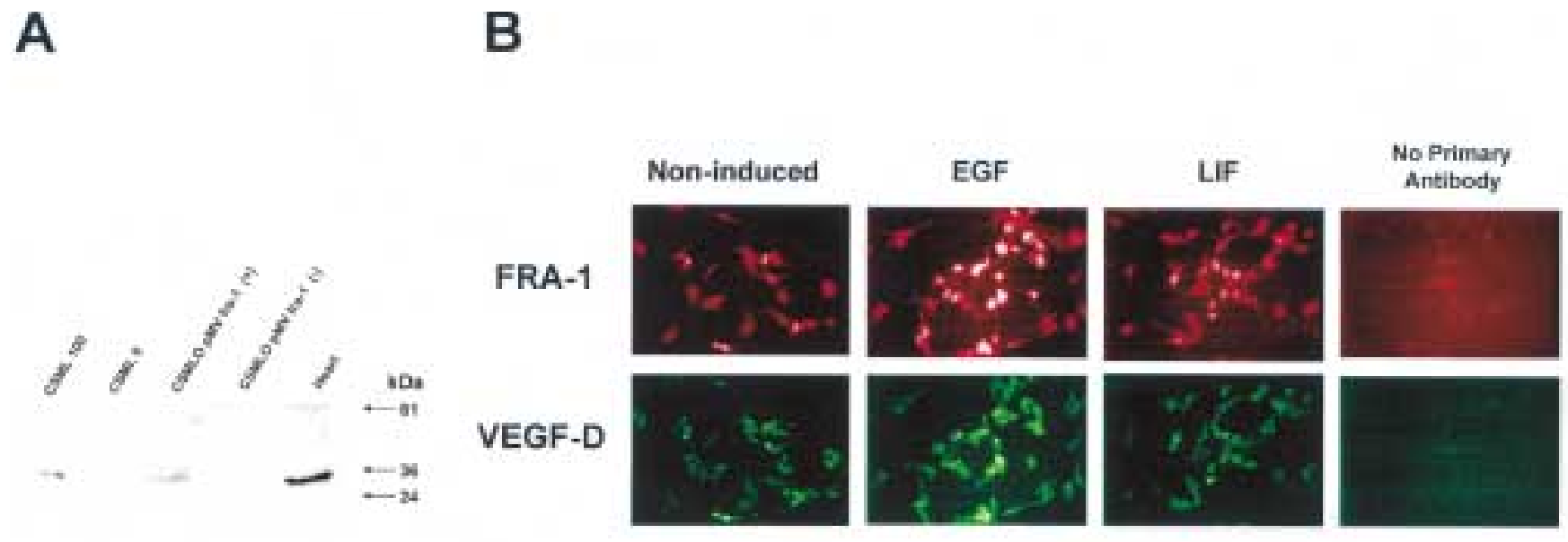

\section{SNB-19}

Fig. 4. VEGF-D and FRA-1 in different stages of malignancy or in growth factor-stimulated cancer cells. (A) Western blot immunoreactivity for VEGF-D in Fra-l (-) or Fra-l (+) cells. Equal amounts (100 $\mu \mathrm{g} / \mathrm{lane})$ of cell lysate proteins derived from CSML0 (low Fra-1), CSML100 (high Fra-1), CSMLO pMV fra-1 (-) (mock-transfected CSML0), and CSMLO pMV fra-1 (+) (fra-1-transfected CSML0) cell lines were electrophoretically separated and blotted onto a PVDF membrane. A positive control lysate from mouse heart was included in the analysis. The immunoreactivity was developed using chemiluminescence. (B) Co-staining for Fra-1 (red) and VEGF-D (green) in SNB-19 cells stimulated with EGF or LIF for $24 \mathrm{hr}$. 
as expected (Mahmood et al., unpublished data), and showed no VEGF-D immunoreactivity, whereas the fra-1 transgene evoked VEGF-D expression in transfected CSML0 cells (Fig. 4A). The size of the detected band was $\sim 33 \mathrm{kDa}$, which corresponds to a form of murine VEGF-D, consisting most probably of the Nterminal pro-peptide and the VEGF homology domain (19) found in VEGF-D-producing organs, such as heart (Fig. 4A), and in VEGF-D-producing cells, such as fibroblasts (5). Thus, Fra- 1 expression turns cells into VEGF-D producers.

\section{Stimulants of AP-1 Activity Evoke an Increase of Fra-1 and VEGF-D in GBM Cells}

To examine preliminarily whether or not the stimulants of AP-1 activity that are highly expressed in GBM are able to increase Fra- 1 and VEGF-D in a sustained manner, we treated SNB-19 GBM cells with EGF and LIF for $24 \mathrm{hr}$ (Fig. 4D). SNB-19 cells were used because of their relatively lower up-regulation of Fra- 1 and VEGF-D when compared with a number of other GBM cell lines (e.g., Fig. 2A). Both EGF and LIF prominently augmented the levels of immunoreactive Fra- 1 and VEGF-D in SNB-19 cells with EGF demonstrating more pronounced effect than LIF, although EGF was used at a higher concentration than LIF (Fig. 4D). The levels of c-Fos remain usually undetectable under those conditions (data not shown).

\section{Discussion}

The most malignant glioma, GBM, strongly expresses VEGF-D, an X-linked c-fos-inducible angiogenic factor. The astrocytoma cell is the primary source of VEGF-D production. We demonstrate that most, if not all, GBM cells produce VEGF-D. The abundance of VEGF-D in GBM appears to be primarily related to the up-regulation of Fra-1, a member of the AP-1 family of transcription factors that have cell-transforming ability, and not to a sustained activation of c-Fos. We propose that the up-regulated angiogenic factors that are associated with oncogenic transformation should be termed onco-angiogens. VEGF-D may thus represent an onco-angiogen in human GBM.

Angiogenic factors that are important in cancer have been linked hypothetically and experimentally to the activation of specific oncogenes, such as Ras (4). Although oncogenesis is a continuous interplay between pro- and anti-proliferative signals, an angiogenic switch permits an uncontrolled proliferation of malignant cells and tumor progression, be it local or metastatic, which is the most important clinical trait of cancer (23). Against this background, VEGF-D has been identified as an oncogene-induced mitogenic factor (5) and a ligand for VEGF receptors 2 and $3(6,7)$. Our data demonstrate that VEGF-D, with its inherently high angiogenic potential, is ubiquitously expressed in the most malignant astroglial neoplasm, GBM, which has long been recognized to be among the most highly vascularized of human tumors (24).
However, the possible activation mechanism(s) of VEGF-D in these brain tumors maybe different than we anticipated. The prototype inducer of VEGF-D, c-Fos protein and its gene, was found to be expressed at low levels, much in contrast to what we found for VEGF-D, but in accordance with the observations made by others on c-fos in astrocytoma cells (e.g., 19). Low c-Fos in cells derived from advanced cancers seems to be a rule rather than exception (25). What is also intriguing and, at first sight, incompatible with relatively low levels of c-Fos is that factors, such LIF, oncostatin M (OSM), and basic fibroblast growth factor (FGF-2) are all constitutively up-regulated in GBM $(26,27$; and data not shown); all these factors induce c-Fos potently, although in a time-restricted manner (20,28,29) (Fig. 5).

These results prompted us to formulate an alternative hypothesis for the cause of an abundance of VEGF-D in GBM. We postulate that VEGF-D is induced by another factor(s) that act through a Fos pathway and are more prominently and/or more stably up-regulated than c-Fos in GBM. Our investigative choice was Fra-l for the following reasons: (1) the gene expression of $\mathrm{fra}^{-1}$ was elevated in

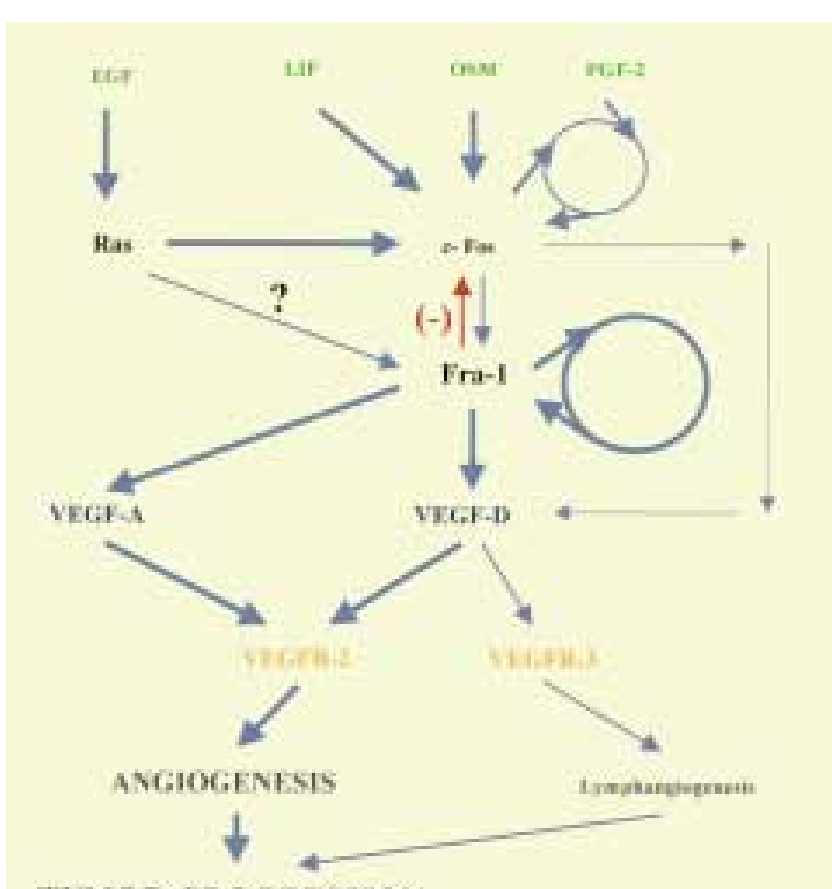

TUMOR PROGRESSION

Fig. 5. Schema of oncogenic activities leading to an abundant expression of VEGFs in GBM. The emphasis is on the activation of the Fos family members and the Fra-1 substituting for the c-Fos. A sustained AP-1 activation is achieved, at least in part, through the positive autoregulatory loops leading to constitutive expression of oncogenic and subsequently angiogenic factors in GBM. An arrow in red represents a negative feedback regulation. Lymphagiogenesis may not take place in GBM. 
GBM in our experiments using cDNA microarrays, (2) Fra-l was found to be a predominant AP-1 component in ras-transformed cells (30), (3) Fra-1 expression was both transforming and tumorigenic (21), and (4) overexpression of Fra-1 was found to be associated with high expression of tissue inhibitor of metalloproteinases-1 (TIMP-1) (22), another Xlinked factor, which is found in a vast majority of patients with GBM (26,31; and data not shown).

Our experiments demonstrate for the first time that Fra- 1 and its partners in transcriptional activation, c-Jun, and to a lesser extent, JunB, are widely expressed in GBM cells. Of importance, we provide both direct and indirect evidence pointing to an association between Fra-1 and VEGF-D. For example, cancer cells that do not express Fra- 1 are deprived of VEGF-D. In a direct experimental approach, expression of a fra-l transgene causes an appearance of VEGF-D in otherwise VEGF-D negative cells. Furthermore, GBM cells stimulated with EGF or LIF for a prolonged period of time demonstrate a significant increase in the levels of Fra-1 and VEGF-D, but not c-Fos. A recent report provides yet another link between Fra- 1 and angiogenesis. In a mouse knockout model, the fra- $1(-/-)$ genotype was lethal due to the lack of proper vascularization of the placenta (32). Our current study suggests that VEGF-D may be a missing angiogenic factor(s) in these mice that contributes to the lethal phenotype.

We bring to light the pathophysiologic importance of AP-1 transcription factors in up-regulating gene expression, or "constitutive" gene expression, in GBM. TIMP-1 and FGF-2, both X-linked, have AP-1 binding sites in their gene promoters $(28,33)$. FGF-2, a mitogenic and angiogenic factor, induces intracellular signaling that is mediated through the AP-1 transcriptional pathway (29). Fra-1 is well positioned to be a stably up-regulated transcription factor in malignancy, because it is under positive control by AP-1 activity (21) (Fig. 5). This transactivating mechanism may be either maintained or amplified in transformed cells. On the other hand, activated Fra-1 negatively regulates c-Fos (21) (Fig. 5). Another AP-1 factor, Fra-2, of similar positive autoregulatory loop of activation to Fra-1 (34), was not detected at significant amounts in GBM (data not shown). Therefore, it is plausible that abundance of LIF, OSM, FGF-2 and other stimulatory signals in GBM, such as EGF, leads to a selection of Fra- 1 as a late and/or long-term mediator of their activated states in astrocytoma cells (Fig. 5). A short-term regulatory transcriptional activation is characteristic of c-Fos (35). For example, serum stimulation evokes rapid expression of c-Fos and JunB; however, this subsides and is followed by a later induction of Fra-1 $(36,37)$. Furthermore, Fra-1 is functionally equivalent to c-Fos (38) and thus Fra-1 can overtake c-Fos-dependent functions (Fig. 5).

Other proteins, which genes possess AP-1 binding sites and have been implicated in the invasiveness and vascularization of GBM, such as urokinase type plasminogen activator (uPA; chromosome 3 ) and gelatinase B (MMP-9; chromosome 20), are constitutively expressed in GBM $(31,39)$. This is again consistent with an unopposed, activated AP-1 axis in GBM and an up-regulation of gene expression in a transcription factor-specific manner (Fig. 5). It is noteworthy that an insufficient expression of uPA and MMP-9 has been suggested to be responsible, at least in part, for the lethal deficiency in vascularization of the placenta in fra-1 (-/-) mice fetuses (32). Furthermore, considering frequent aneuploidy of chromosome $\mathrm{X}$ in GBM cells, we cannot exclude a contributing effect of gene duplication on VEGF-D and other factors overexpression.

However, the mechanism(s) responsible for a frequent appearance of X-linked CTAs in human malignancies remains to be unraveled, because those tumor antigens, such MAGE, GAGE, and PAGE-1, do not have AP-1 binding sites within their promoters. Our current study indicates different from an AP-1 activation abnormality that occurs in transformed cells and induces expression of CTAs. This abnormality must be very specific to the $\mathrm{X}$ chromosome.

It has previously been documented that VEGF-A and VEGF receptor 2 (Flk-1) are present in GBM and this has direct implications for brain tumor pathogenesis $(40,41)$. Preclinical therapeutic attempts at blocking VEGF receptor 2 were successful in various models of cancer (42-44). However, a recent study demonstrated that neutralization of VEGF-A alone is insufficient for sustained anti-tumor activity in brain tumors (45). Nor is Ras oncogene-induced VEGF-A expression sufficient to promote aggressive tumor growth (46) (Fig. 5). Our study provides evidence that VEGF-D may be one of the reasons for (1) an incomplete and short-lived efficacy of anti-tumor approaches based on blocking selected individual vasoactive agents, such as VEGF-A and (2) an oncogeneevoked tumor vascularization and progression.

In summary, we identify an angiogenic factor, VEGF-D, that is ubiquitous in highly vascularized and locally invasive brain tumors. We provide evidence for the involvement of the Fos family of transcription factors in this phenomenon, with Fra-1 being the preeminent member (Fig. 5). Our schema illustrates pathways of possible interactions involved in new blood vessel formation and endothelial cell proliferation in GBM (Fig. 5). Recent data further support an important role of VEGF-D in tumor progression. It has been documented that a lymphangiogenic activity of VEGF-D, besides an angiogenic one, enables a metastatic spread of cancer through the lymphatics (47) (Fig. 5). The data we present here emphasize a key role for AP-1 regulation of a specific subset of genes and possible selfsustainable loops of activation of factors important in GBM pathogenesis (Fig. 5). However, recent data indicate that other cancers, such as breast, thyroid, and skin cancers $(22,48,49)$ may also utilize these mechanisms, particularly in more advanced stages 
of disease. Importantly, it seems now that the basal overexpression of VEGF-A maybe due to an increased AP- 1 activity in some cancers. This is because the gene for VEGF-A also contains AP-1 binding sites (Fig. 5) and the head and neck cancer has been recently shown to have a concomitant up-regulation of VEGF-A that is associated with co-activation of AP-1 factors, such as Fra-l and c-Jun (50). By this fact, transcription factors, such as Fra- 1 and c-Jun, actually become "oncogenic." An unopposed, activated AP- 1 axis is thus a hallmark of GBM and may represent a marker of the disease, a role similar to play by plasma membrane receptors (8). In view of our current results, the VEGF-D system and AP-1 activity appear to be very attractive targets for new rational molecular anti-cancer therapies.

\section{Acknowledgments}

The American Cancer Society (W.D.) supported this work. We thank all neurosurgeons in the Section of Neurosurgery at Hershey who helped to secure tumor samples, especially Dr. Stephen K. Powers. We thank Dr. Stephen B. Baylin for comments on the manuscript. M.G.A. and S.A.S. were supported by the National Health and Medical Research Council of Australia and the Anti-Cancer Council of Victoria. E.T. was supported by the Danish Cancer Society.

\section{References}

1. Salcman M. (1993). Concepts in neurosurgery: neurobiology of brain tumors. Baltimore, MD: Williams \& Wilkins.

2. Long DM. (1970). Capillary ultrastructure and the blood-brain barrier in human malignant brain tumors. J. Neurosurg. 32: 127-144.

3. Goldman CK, Kim J, Wong W-L, King V, Brock T, Gillespie GY. (1993). Epidermal growth factor stimulates vascular endothelial growth factor production by human malignant glioma cells: a model of glioblastoma multiforme pathophysiology. Mol. Biol. Cell 4: 121-133.

4. Kerbel RS, Viloria-Petit A, Okada F, Rak J. (1998). Establishing a link between oncogenes and tumor angiogenesis. Mol. Med. 4: 286-295.

5. Orlandini M, Larconcini L, Ferruzzi R, Oliviero S. (1996). Identification of a c-fos-induced gene that is related to the platelet-derived growth factor/vascular endothelial growth factor family. Proc. Natl. Acad. Sci. U.S.A. 93: 11675-11680.

6. Achen MG, Jeltsch M, Kukk E, et al. (1998). Vascular endothelial growth factor D (VEGF-D) is a ligand for the tyrosine kinases VEGF receptor 2 (Flkl) and VEGF receptor 3 (Flt4). Proc. Natl. Acad. Sci. U.S.A. 95: 548-553.

7. Marconcini L, Marchio S, Morbidelli L, et al. (1999). c-fosinduced growth factor/vascular endothelial growth factor D induces angiogenesis in vivo and in vitro. Proc. Natl. Acad. Sci. U.S.A. 96: 9671-9676.

8. Debinski W, Gibo D, Hulet SW, Connor JR, Gillespie GY. (1999). Receptor for interleukin 13 is a marker and therapeutic target for human high-grade glioma Clin. Cancer Res. 5: 985990.

9. Debinski W, Gibo D, Obiri N, Kealiher A, Puri R. (1998). Novel anti-brain tumor cytotoxins specific for cancer cells. Nature Biotech. 16: 449-453.

10. Debinski W, Gibo DM. (2000). Molecular expression analysis of restrictive receptor for interleukin 13, a brain tumorassociated cancer/testis antigen. Mol. Med. 6: 440-449.
11. Debinski W, Slagle B, Gibo DM, Powers SK, Gillespie GY. (2000). Expression of a restrictive receptor for interleukin 13 is associated with glial transformation. J. Neurooncol. 48: 103111.

12. Old LJ, Chen TC. (1998). New paths in human cancer serology. J. Exp. Med. 187: 1163-1167.

13. Mintz A, Debinski W. (2000). Cancer genetics/epigenetics and the $\mathrm{X}$ chromosome: Possible new links for malignant glioma pathogenesis and immune-based therapy. Crit. Rev. Oncogen. 11: 77-95.

14. Jenkins NA, Woolatt E, Crawford J, et al. (1997). Mapping of the gene for vascular endothelial growth factor-D in mouse and man to the $\mathrm{X}$ chromosome. Chromosome Res. 5: 502-505.

15. Achen MG, Roufail S, Domagala T, et al. (2000). Monoclonal antibodies to vascular endothelial growth factor-D block its interaction with both VEGF receptor-2 and VEGF receptor-3. Eur. J. Biochem. 267: 2505-2515.

16. Chomczynski P, Sacchi N. (1987). Single-step method of RNA isolation by acid guanidinium thiocyanate-phenol-chloroform extraction. Anal. Biochem. 162: 156-159.

17. Rocchigiani M, Lestingi M, Luddi A, et al. (1998). Human FIGF: cloning, gene structure, and mapping to chromosome Xp22.1 between the PIGA and the GRPR genes. Genomics 47: 207-2 16.

18. McKeever PE. (1998). Insights about brain tumors gained through immunohistochemistry and in situ hybridization of nuclear and phenotypic markers. J. Histochem. Cytochem. 46: 585594.

19. Stacker SA, Stenvers K, Caesar C, et al. (1999) Biosynthesis of vascular endothelial growth factor-D involves proteolytic processing which generates non-covalent homodimers. J. Biol. Chem. 274: 32127-32136.

20. Halfter H, Kremerskothen J, Weber J, et al. (1998). Growth inhibition of newly established human glioma cell lines by leukemia inhibitory factor. J. Neurooncol. 39: 1-18.

21. Bergers G, Graninger P, Braselmann S, Wrighton C, Busslinger M. (1995). Transcriptional activation of the fra-1 gene by AP-1 is mediated by regulatory sequences in the first intron. Mol. Cell. Biol. 15: 3748-3758.

22. Kustikova O, Kramerov D, Grigorian M, et al. (1998). Fra-1 induces morphological transformation and increases in vitro invasiveness and motility of epithelioid adenocarcinoma cells. Mol. Cell. Biol. 18: 7095-7105.

23. Folkman J. (1995). Angiogenesis in cancer, vascular, rheumatoid and other diseases. Nat. Med. 1: 27-31.

24. Brem S, Cotran R, Folkman J. (1972). Tumor angiogenesis: a quantitative method for histologic grading. J. Natl. Cancer Inst. 48: $347-356$.

25. Tulchinsky E. (2000). Fos family members: regulation, structure, and role in oncogenic transformation. Histol. Histopathol. 15: $921-928$.

26. Lilja A, Stanisic M, Salford L. (2000). Abnormal expression of OSM, CNTF and LIF in human brain tumors. Neuro-Oncology 2: S22.

27. Zagzag D, Miller DC, Sato Y, Rifkin DB, Burstein DE. (1990). Immunohistochemical localization of basic fibroblast growth factor in astrocytomas. Cancer Res. 50: 7393-7398.

28. Botelho FM, Edwards DR, Richards CD. (1998). Oncostatin M stimulates c-Fos to bind a transcriptionally responsive AP-1 element within the tissue inhibitor of metalloproteinase-1 promoter. J. Biol. Chem. 273: 5211-5218.

29. Mohan R, Sivak J, Ashton P, et al. (2000). Curcuminoids inhibit the angiogenic response stimulated by fibroblast growth factor-2, including expression of matrix metalloproteinase gelatinase B. J. Biol. Chem. 275: 10405-10412.

30. Mechta F, Lallemand D, Pfarr CM, Yaniv M. (1997). Transformation by ras modifies API composition and activity. Oncogene 14: 837-847.

31. Nakano A, Tani E, Miyazaki K, Yamamoto Y, Furuyama J-I. (1995). Matrix metalloproteinases and tissue inhibitors of metalloproteinases in human gliomas. J. Neurosurg. 83: 298-307.

32. Schreiber M, Wand Z-Q, Jochum W, Fetka I, Elliott C, Wagner EF. (2000). Placental vascularisation requires the AP-1 component Fra-1. Development 127: 4937-4948. 
33. Phillips BW, Sharma R, Leco PA, Edwards DR. (1999). A sequence-selective single-strand DNA-binding protein regulates basal transcription of the murine tissue inhibitor of metalloproteinases-1 (Timp-1) gene. J. Biol. Chem. 274: 22 19722207.

34. Murakami M, Motoyasu U, Iba H. (1999). Fra-2-positive autoregulatory loop triggered by mitogen-activated protein kinase (MAPK) and Fra-2 phosphorylation sites by MAPK. Cell Growth Differ. 10: 333-342.

35. Ransone LJ, Verma IM. (1990). Nuclear proto-oncogenes fos and jun. Annu. Rev. Cell Biol. 6: 539-557.

36. Kovary K, Bravo R. (1992). Existence of different Fos/Jun complexes during the G0-to-Gl transition and during exponential growth in mouse fibroblasts: differential role of Fos proteins. Mol. Cell. Biol. 12: 5015-5023.

37. Gruda MC, Kovary K, Metz R, Bravo R. (1994). Regulation of Fra- 1 and Fra-2 phosphorylation differs during the cell cycle of fibroblasts and phosphorylation in vitro by MAP kinase affects DNA binding activity. Oncogene 9: 2537-2547.

38. Fleischmann A, Hafezi F, Elliott C, Reme CE, Ruther U, Wagner EF. (2000). Fra-1 replaces c-Fos-dependent functions in mice. Genes Dev. 14: 2695-2700.

39. Mishima K, Mazar AP, Gown A, et al. (2000). A peptide derived from the non-receptor binding region of urokinase plasminogen activator inhibits glioblastoma growth and angiogenesis in vivo in combination with cisplatin. Proc. Natl. Acad. Sci. U.S.A. 97: 8484-8489.

40. Millauer B, Shawver LK, Plate KH, Risau W, Ullrich A. (1994). Glioblastoma growth inhibited in vivo by a dominant-negative Flk-1 mutant. Nature 367: 576-579.

41. Takano S, Yoshii Y, Kondo S, et al. (1996). Concentrations of vascular endothelial growth factor in the serum and tumor tissue of brain tumor patients. Cancer Res. 56: 2185-2190.
42. Strawn LM, McMahon G, App H, et al. (1996) Flk-1 as a target for tumor growth inhibition. Cancer Res. 56: 3540-3545.

43. Skobe M, Rockwell P, Goldstein N, Vosseler S, Fusenig NE. (1997). Halting angiogenesis suppresses carcinoma cell invasion. Nat. Med. 3: 1222-1227.

44. Prewett M, Huber J, Li Y, et al. (1999) Anti-vascular endothelial growth factor receptor (Fetal Liver Kinase1) monoclonal antibody inhibits tumor angiogenesis and growth of several mouse and human tumors. Cancer Res. 59: 5209-5218.

45. Rubenstein JL, Kim J, Ozawa T, et al. (2000). Anti-VEGF antibody treatment of glioblastoma prolongs survival but results in increased vascular cooption. Neoplasia 2: 306-314.

46. Okada F, Rak J, St. Croix B, et al. (1998). Impact of oncogenes in tumor angiogenesis: mutant $\mathrm{K}$-ras up-regulation of vascular endothelial growth factor/vascular permeability factor is necessary, but not sufficient for tumorigenicity of human colorectal carcinoma cells. Proc. Natl. Acad. Sci. U.S.A. 95: 3609-3614.

47. Stacker SA, Caesar C, Baldwin ME, et al. (2001). VEGF-D promotes the metastatic spread of tumor cells by the lymphatics. Nat. Med. 7: 168-175.

48. Battista S, de Nigris F, Fedele M, et al. (1998). Increase in AP-1 activity is a general event in thyroid cell transformation in vitro and in vivo. Oncogene 17: 377-385.

49. Zoumpourlis V, Papassava P, Lindardopoulos S, Gillespie D, Balmain A, Pintzas A. (2000). High levels of c-Jun, Fra-1, Fra-2 and ATF-2 proteins correlate with malignant phenotypes in the multistage mouse skin carcinogenesis model. Oncogene 19: 4011-4021.

50. Bancroft CC, Chen Z, Dong G, et al. (2001). Coexpression of proangiogenic factors IL-8 and VEGF by human head and neck squamous cell carcinoma involves coactivation by MEK-MAPK and IKK-NF-kB signal pathways. Clin. Cancer Res. 7: 435-442. 